\title{
AVALIAÇÃO DA ORGANIZAÇÃO DO CUIDADO EM SAÚDE MENTAL NA ATENÇÃO BÁSICA À SAÚDE DO BRASIL
}

\author{
EVALUATION OF THE ORGANIZATION OF CARE IN MENTAL \\ HEALTH IN PRIMARY HEALTH CARE IN BRAZIL \\ EVALUACIÓN DE LA ORGANIZACIÓN DEL CUIDADO DE LA SALUD \\ MENTAL EN LA ATENCIÓN BÁSICA DE LA SALUD EN BRASIL
}

Tiziana Bezerra Gerbaldo ${ }^{1}$

\author{
Adenilda Teixeira Arruda ${ }^{2}$ \\ Bernardo Lessa Horta ${ }^{3}$ \\ Luiza Garnelo ${ }^{4}$
}

Resumo Este artigo resultou de um estudo transversal sobre o cuidado em saúde mental com 29.778 equipes da Estratégia Saúde da Família de todo o Brasil (87,1\% do total), incluindo avaliação normativa dos dados do segundo ciclo do Programa Nacional de Melhoria do Acesso e da Qualidade da Atenção Básica (2013-2014) nas dimensões perfil dos profissionais, promoção da saúde mental, gestão e oferta do cuidado. Os resultados mostraram que $33,8 \%$ dos entrevistados tinham vínculo trabalhista precário e $60,3 \%$ deles se sentiam despreparados para atuar em saúde mental. A oferta do conjunto de ações avaliadas só ocorreu em 9,5\% das equipes em todo o Brasil (2,2\% na região Norte). Cerca de metade não desenvolvia estratégias de promoção da saúde e apenas 9,8\% efetivavam a gestão do cuidado. Concluiu-se que os baixos percentuais de implantação em nível nacional coexistem com expressivas desigualdades regionais, com piores resultados no Norte. Fazem-se necessários o fortalecimento de ações de promoção da saúde, a qualificação das equipes, a desprecarização de vínculos e o reordenamento da gestão do cuidado.

Palavras-chave saúde mental; atenção básica; programas de saúde.

\begin{abstract}
The article is the result of a cross-sectional study about care in mental health with 29,778 teams from the Family Health Strategy from all over Brazil ( $87.1 \%$ of the total of teams), including a normative evaluation of the data of the second cycle of the National Program for the Enhancement of Access and Quality in Primary Health Care (2013-2014) in the dimensions of the profile of the professionals, promotion of mental health, management, and offer of care. The results showed that $33.8 \%$ of the interviewees had precarious labor relationships, and that $60.3 \%$ of them did not feel prepared to work in mental health. The offer of the set of actions evaluated only occurred in $9.5 \%$ of the teams in all of Brazil (2.2\% in the Northern region). Around half of them did not develop strategies to promote health, and only $9.8 \%$ actually carried out the management of care. We concluded that the low percentages of implementation on the national level coexist with significant regional inequalities, with the worst results coming from the Northern region. It is necessary to strengthen the actions of promotion of health, the qualification of the teams, to reverse the precariousness of the labor relationships, and to reorganize the management of care.
\end{abstract}

Keywords mental health; primary health care; health programs.

(cc) BY Este é um artigo publicado em acesso aberto sob uma licença Creative Commons. 


\section{Introdução}

De acordo com a Organização Mundial da Saúde (OMS), os transtornos mentais são importante causa de morbidade e de mortalidade prematura, representando $12 \%$ da carga mundial de doença (World Health Organization, 2008). No Brasil, estimativas do Ministério da Saúde apontam que 3\% da população necessita de cuidados contínuos em saúde mental e $9 \%$ de atendimento eventual, no caso de transtornos de menor gravidade (Brasil, 2003).

A rede de atenção básica (AB) é o nível preferencial para oferta de ações de saúde mental e ponto estratégico da Rede de Atenção Psicossocial (RAPS) (Brasil, 2011, 2012). O cuidado em saúde mental na $A B$ envolve um conjunto de atividades que visam ao controle de sintomas, à prevenção de recidivas e à redução do risco de internação, envolvendo diagnóstico precoce, tratamento - incluído o manejo de psicotrópicos - e acompanhamento adequados, além de promoção da saúde (World Health Organization, 2001).

Entre os benefícios dos cuidados de saúde mental na atenção básica, são apontados desfechos clínicos positivos, melhora da qualidade de vida de pacientes com depressão (Schoenbaum, 2002), ampliação da cobertura assistencial dos agravos mentais e redução das práticas manicomiais (Rodrigues e Moreira, 2012).

No que diz respeito à prevalência de transtornos mentais entre os usuários do sistema de saúde, publicações mais recentes que avaliaram a prevalência de transtornos mentais comuns em usuários da atenção básica nos municípios de São João del Rei (MG) e São Paulo (SP) encontraram prevalências de 24,95\% e 43,7\%, respectivamente, em usuários da Estratégia Saúde da Família (ESF), indicando a importância do atendimento a tais problemas na rede de atenção primária (Moreira et al., 2011; Maragno et al., 2006).

$\mathrm{O}$ estudo que originou este artigo procurou avaliar a organização do cuidado (Cecílio, 2011) em saúde mental desenvolvido na ESF no Brasil por meio das dimensões promoção da saúde mental, gestão e oferta do cuidado. Investigaram-se variáveis como organização e disponibilidade de cuidados em saúde mental na Estratégia Saúde da Família, planejamento das ações e definição de fluxos e rotinas no atendimento aos usuários. Os dados provêm do segundo ciclo avaliativo do Programa Nacional de Melhoria do Acesso e da Qualidade da Atenção Básica (PMAQ-AB), que objetiva incentivar a melhoria da qualidade e ampliação do acesso, efetividade e qualidade das ações ofertadas na rede básica de saúde (Brasil, 2013). A avaliação realizada se caracterizou como uma apreciação normativa, nos termos de Champagne e colaboradores (2011). 


\section{Desenho do estudo}

O estudo 5 que deu origem a este artigo foi do tipo transversal, desenvolvido com base nas informações obtidas no banco de dados do componente de avaliação externa do segundo ciclo do PMAQ- $\mathrm{AB}$ - avaliação esta realizada por instituições de ensino e pesquisa em 2013-2014. A coleta dos dados foi feita de forma eletrônica, utilizando-se equipamentos do tipo tablet. Foram entrevistadas 29.778 equipes da Estratégia Saúde da Família (eSFs) que atuavam na $A B$, equivalendo a $87,1 \%$ do total de 34.185 equipes implantadas no país (Brasil, 2015). Dadas as características do programa PMAQ-AB, a seleção de equipes foi feita por adesão voluntária ao processo avaliativo.

O instrumento de coleta de dados contemplava perguntas sobre o perfil dos profissionais da ESF e dos serviços ofertados nas unidades de saúde da família. O bloco relativo aos serviços constava de quatro módulos: observação na unidade básica de saúde; entrevista com profissional de saúde e verificação de documentos; entrevista com os usuários; entrevista com o Nasf (Brasil, 2013). Para este artigo foi analisado o segundo módulo, nos itens II.23 (atenção ao usuário em sofrimento psíquico); II.3, II.4, II.5 e II.7 (utilizados para avaliar o perfil profissional e a qualificação dos entrevistados); itens II.12, II.14 e II.23 (gestão do cuidado e acolhimento à demanda espontânea) e II.26 (ações de promoção à saúde). Do conjunto desses itens foram selecionadas seis perguntas relativas ao perfil profissional e 18 relativas às ações ofertadas ao usuário em sofrimento psíquico, acolhimento à demanda e promoção da saúde.

O plano de análise contemplou o reagrupamento das perguntas oriundas do banco, selecionadas segundo sua congruência com as dimensões avaliativas priorizadas no estudo (perfil profissional, promoção da saúde, oferta de cuidado em saúde mental e gestão do cuidado em saúde mental). A seleção e o reagrupamento das variáveis relativas às ações em saúde mental tomaram como base a portaria n. 3.088 (Brasil, 2011), que preconiza as atividades a serem desenvolvidas nas unidades básicas de saúde. $\mathrm{O}$ conjunto de dimensões e subdimensões avaliativas está disposto no Quadro 1.

Todas as equipes cujas respostas constavam no banco da avaliação externa foram incluídas, já que não houve perdas.

\begin{tabular}{|ll|}
\hline Quadro 1 \\
\hline $\begin{array}{l}\text { Descrição das dimensões e subdimensões de avaliação da oferta do cuidado em saúde mental } \\
\text { Dimensões }\end{array}$ \\
\hline de análise & Subdimensóes (variáveis oriundas do questionário) \\
& II.3.2. Profissão \\
& II.3.5. Tempo de trabalho na equipe \\
Perfil & II.4.1 Possui ou está em formação complementar? \\
& II.5.2. Qual seu tipo de vínculo? \\
& II.7.1. Participa de ações de educação permanente organizadas pela gestão municipal? \\
\hline
\end{tabular}


Continuação - Quadro 1

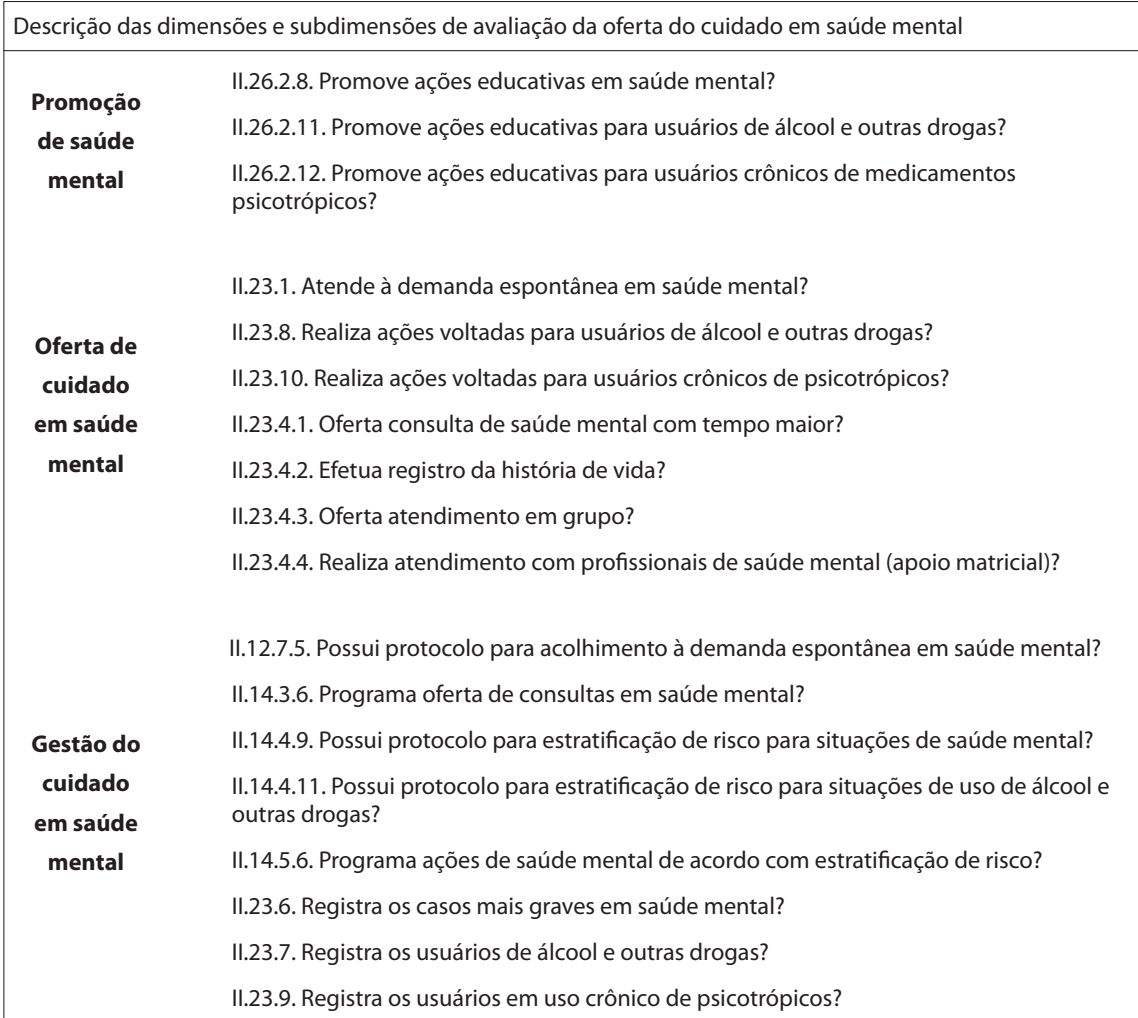

Fonte: Adaptado de Instrumento de avaliação externa para as equipes de atenção básica.

Ver em: <http://189.28.128.100/dab/docs/portaldab/documentos/instrumento_ae_sfp.pdf>.

Ao contrário de outras formas de atenção programática, como a atenção ao pré-natal e o controle de doenças transmissíveis (Brasil, 2015), que dispõem de protocolos clínicos e indicadores que definem padrões mínimos de qualidade do cuidado e nível de resolutividade desejável na atenção básica, não foram encontrados parâmetros equivalentes para atenção à saúde mental. Tal circunstância limitou a avaliação às questões previamente definidas no questionário do PMAQ, impedindo que se avaliasse a adequação das ações ofertadas na ESF.

Os dados foram tabulados em planilha do programa Microsoft Office Excel 2013. Para análise de dados, utilizou-se o software IBM Statistical Package for Social Science (SPSS) versão 22. As ações foram investigadas individualmente e por dimensões, contabilizando-se a quantidade de ações realizadas por grupo de análise e agrupando-as em intervalos. A análise por dimensão considerou apenas as respostas que indicavam execução da ação investigada. Além da análise descritiva, realizou-se análise bivariada para avaliar a associação de cada componente selecionado com as regiões geopolíticas brasileiras. As diferenças na distribuição das características do cuidado em saúde mental para 
cada região do país foram verificadas com o teste qui-quadrado, adotando um nível de significância de 5\%.

A pesquisa foi aprovada pelo Comitê de Ética em Pesquisa em Seres Humanos da Escola Nacional de Saúde Pública Sergio Arouca (Ensp) da Fundação Oswaldo Cruz (Fiocruz) e recebeu o parecer n. 357.974 em 15/08/2013.

\section{Análise dos resultados}

A maioria das entrevistas foi realizada com os enfermeiros $(93,4 \%)$ com até um ano de atuação na equipe $(47,5 \%)$. A proporção de indivíduos com menos de um ano de atuação foi maior nas regiões Norte e Centro-Oeste. Com relação ao vínculo, 40,4\% do total de entrevistados eram servidores públicos estatutários, com maior percentual na região Sul $(58,7 \%)$. Nas regiões Norte e Nordeste foram observados maiores percentuais de entrevistados com contratos temporários, $51,9 \%$ e $43,1 \%$, respectivamente, enquanto na região Sul apenas $10 \%$ dos entrevistados se enquadravam nesta alternativa.

Com relação à formação complementar, 83,6\% declararam possuir algum tipo de qualificação adicional, sendo que $30 \%$ dos enfermeiros informaram ter especialização em saúde da família e 18,2\% em saúde pública. A grande maioria $(88,9 \%)$ dos entrevistados participava de ações de educação permanente organizadas pelo sistema municipal, cujas temáticas não foram investigadas pelo instrumento. Com relação às demandas de saúde mental, cerca de quatro em cada dez entrevistados declararam não se sentir preparados para lidar com esses casos (Tabela 1).

Tabela 1

Perfil dos profissionais das equipes de atenção básica entrevistados no PMAQ-AB segundo ciclo. Brasil e regiões, $2013 / 2014$

\begin{tabular}{|c|c|c|c|c|c|c|c|}
\hline \multirow[b]{2}{*}{ Variável } & \multirow[b]{2}{*}{ Brasil } & \multicolumn{5}{|c|}{ Região } & \multirow[b]{2}{*}{ p-valor } \\
\hline & & Norte & Nordeste & Sudeste & Sul & Centro-Oeste & \\
\hline Profissão & & & & & & & $<0,001$ \\
\hline Médico & 5,7 & 8,3 & 3,3 & 7,2 & 6,7 & 5,5 & \\
\hline Enfermeiro & 93,4 & 91,0 & 96,1 & 92,0 & 91,5 & 93,0 & \\
\hline Outro nível superior & 0,9 & 0,7 & 0,6 & 0,8 & 1,8 & 1,4 & \\
\hline Tempo de trabalho & & & & & & & $<0,001$ \\
\hline \multicolumn{8}{|l|}{ na equipe } \\
\hline Até um ano & 47,5 & 53,4 & 49,8 & 48,1 & 36,0 & 51,7 & \\
\hline De 2 a 5 anos & 29,3 & 23,8 & 27,2 & 34,1 & 25,0 & 31,4 & \\
\hline De 6 a 9 anos & 10,2 & 8,3 & 12,0 & 9,6 & 9,2 & 8,3 & \\
\hline 10 anos ou mais & 5,2 & 4,2 & 6,0 & 4,6 & 5,2 & 4,6 & \\
\hline Não sabe & 7,8 & 10,4 & 5,0 & 3,5 & 24,6 & 3,9 & \\
\hline
\end{tabular}


Perfil dos profissionais das equipes de atenção básica entrevistados no PMAQ-AB segundo ciclo. Brasil e regiões, 2013/2014

\begin{tabular}{|c|c|c|c|c|c|c|c|}
\hline \multirow[b]{2}{*}{ Variável } & \multirow[b]{2}{*}{ Brasil } & \multicolumn{5}{|c|}{ Região } & \multirow[b]{2}{*}{ p-valor } \\
\hline & & Norte & Nordeste & Sudeste & Sul & Centro-Oeste & \\
\hline Vínculo & & & & & & & $<0,001$ \\
\hline Estatutário & 40,4 & 35,6 & 41,7 & 29,3 & 58,7 & 51,6 & \\
\hline Empregado CLT & 7,0 & 1,8 & 3,4 & 7,3 & 20,2 & 1,9 & \\
\hline Contrato CLT & 14,7 & 4,9 & 6,7 & 30,1 & 8,0 & 5,9 & \\
\hline Contrato temporário & 33,8 & 51,9 & 43,1 & 30,1 & 10,0 & 36,2 & \\
\hline Outros & 4,1 & 5,8 & 5,1 & 3,1 & 3,1 & 4,4 & \\
\hline \multicolumn{8}{|l|}{ Possui formação } \\
\hline complementar & 83,6 & 77,7 & 87,3 & 82,2 & 83,7 & 77,5 & $<0,001$ \\
\hline \multicolumn{8}{|l|}{ Participa de educação } \\
\hline permanente & 88,9 & 86,3 & 87,5 & 91,8 & 88,7 & 84,8 & $<0,001$ \\
\hline \multicolumn{8}{|l|}{ Tem preparo para lidar com } \\
\hline demandas em saúde mental & 39,7 & 26,7 & 34,1 & 45,0 & 49,3 & 35,6 & $<0,001$ \\
\hline $\mathrm{N}$ & 29.778 & 2.160 & 10.768 & 10.100 & 4.509 & 2.241 & \\
\hline
\end{tabular}

Fonte: Banco de dados do PMAQ-AB, segundo ciclo, 2013/2014.

Ver em: $<$ http://dab.saude.gov.br/portaldab/ape_pmaq.php?conteudo=2_ciclo $>$.

A Tabela 2 mostra os resultados referentes à promoção da saúde, oferta e gestão do cuidado. As ações educativas e de promoção da saúde apresentaram baixos percentuais de respostas, variando de $23,5 \%$ para a promoção e educação para usuários de medicamentos psicotrópicos a 33,9\% para usuários de álcool e outras drogas. A se destacar que nessas ações a região Norte também apresentou os menores percentuais de atividades desenvolvidas, enquanto as maiores frequências foram observadas na região Sul. A maioria das equipes afirmou atender pessoas em sofrimento psíquico na unidade $(88,2 \%)$, mas somente metade $(55,2 \%)$ programava a oferta de consultas para esse público e realizava ações para usuários crônicos de medicamentos psicotrópicos $(48,8 \%)$. Com relação ao atendimento com profissionais de saúde mental do Nasf ou outro tipo de apoio matricial, 62,0\% das equipes afirmaram executar essa atividade. Para todas as outras ações, o percentual não alcançou $50 \%$. A oferta de atendimento em grupo foi a estratégia menos utilizada pelas equipes $(27,6 \%)$, sendo realizada apenas por 9,0\% das equipes entrevistadas na região Norte. Na gestão do cuidado, somente a oferta programada de consultas em saúde mental e o registro dos casos mais graves e de usuários de medicamentos psicotrópicos ultrapassaram $50 \%$ das respostas para o país como um todo. A região Norte persistiu com os menores índices para todas as ações. 
Tabela 2

Dimensões e variáveis estudadas na organização e gestão do cuidado em saúde mental pelas equipes de saúd da família entrevistadas no PMAQ-AB segundo ciclo. Brasil e regiões, 2013/2014

\begin{tabular}{|c|c|c|c|c|c|c|c|}
\hline \multirow[b]{2}{*}{ Dimensões } & \multicolumn{7}{|c|}{ Região } \\
\hline & Brasil & Norte & Nordeste & Sudeste & Sul & $\begin{array}{l}\text { Centro- } \\
\text { Oeste }\end{array}$ & p-valor \\
\hline \multicolumn{8}{|l|}{ Promoção de saúde mental } \\
\hline Promove ações educativas em saúde mental? & 32,4 & 24,7 & 29,9 & 33,9 & 40,4 & 28,7 & $<0,001$ \\
\hline $\begin{array}{l}\text { Promove ações educativas para } \\
\text { usuários de álcool e outras drogas? }\end{array}$ & 33,9 & 32,1 & 35,7 & 30,9 & 37,3 & 33,2 & $<0,001$ \\
\hline $\begin{array}{l}\text { Promove ações educativas para usuários } \\
\text { crônicos de medicamentos psicotrópicos? }\end{array}$ & 23,5 & 16,2 & 23,7 & 23,7 & 27,1 & 21,9 & $<0,001$ \\
\hline \multicolumn{8}{|l|}{ Oferta de cuidado em saúde mental } \\
\hline $\begin{array}{l}\text { Atende à demanda espontânea } \\
\text { em saúde mental? }\end{array}$ & 88,2 & 72,4 & 85,2 & 92,3 & 96,1 & 83,2 & $<0,001$ \\
\hline $\begin{array}{l}\text { Realiza ações voltadas para usuários } \\
\text { de álcool e outras drogas? }\end{array}$ & 36,6 & 25,0 & 37,0 & 38,6 & 41,0 & 28,2 & $<0,001$ \\
\hline $\begin{array}{l}\text { Realiza ações voltadas para usuários } \\
\text { crônicos de psicotrópicos? }\end{array}$ & 48,8 & 32,7 & 49,3 & 51,7 & 53,0 & 40,6 & $<0,001$ \\
\hline $\begin{array}{l}\text { Oferta consulta de saúde mental } \\
\text { com tempo maior? }\end{array}$ & 47,3 & 30,7 & 45,1 & 51,9 & 55,3 & 37,0 & $<0,001$ \\
\hline Oferta atendimento em grupo? & 27,6 & 9,0 & 19,1 & 36,7 & 41,2 & 18,5 & $<0,001$ \\
\hline $\begin{array}{l}\text { Realiza atendimento com profissionais } \\
\text { de saúde mental (apoio matricial)? }\end{array}$ & 62,0 & 38,1 & 62,1 & 68,9 & 67,8 & 41,9 & $<0,001$ \\
\hline Efetua registro da história de vida? & 45,2 & 28,1 & 41,7 & 52,3 & 51,7 & 33,2 & $<0,001$ \\
\hline \multicolumn{8}{|l|}{ Gestão do cuidado em saúde mental } \\
\hline $\begin{array}{l}\text { Possui protocolo para acolhimento à } \\
\text { demanda espontânea em saúde mental? }\end{array}$ & 47,1 & 23,7 & 41,3 & 62,6 & 45,5 & 31,1 & $<0,001$ \\
\hline $\begin{array}{l}\text { Possui protocolo para estratificação de } \\
\text { risco para situações de saúde mental? }\end{array}$ & 49,6 & 29,2 & 45,5 & 63,2 & 46,2 & 34,0 & 0,001 \\
\hline $\begin{array}{l}\text { Possui protocolo para estratificação de } \\
\text { risco para situações de uso de álcool e } \\
\text { outras drogas? }\end{array}$ & 33,4 & 15,8 & 28,5 & 43,8 & 35,5 & 22,1 & $<0,001$ \\
\hline $\begin{array}{l}\text { Programa oferta de consultas em } \\
\text { saúde mental? }\end{array}$ & 55,2 & 49,6 & 52,4 & 58,9 & 59,9 & 48,4 & $<0,001$ \\
\hline
\end{tabular}


Continuação - Tabela 2

Dimensões e variáveis estudadas na organização e gestão do cuidado em saúde mental pelas equipes de saúde da família entrevistadas no PMAQ-AB segundo ciclo. Brasil e regiões, 2013/2014

\begin{tabular}{|c|c|c|c|c|c|c|c|}
\hline \multirow[b]{2}{*}{ Dimensões } & \multicolumn{7}{|c|}{ Região } \\
\hline & Brasil & Norte & Nordeste & Sudeste & Sul & $\begin{array}{l}\text { Centro- } \\
\text { Oeste }\end{array}$ & p-valor \\
\hline \multicolumn{8}{|l|}{ Gestão do cuidado em saúde mental } \\
\hline $\begin{array}{l}\text { Programa ações de saúde mental de acordo } \\
\text { com estratificação de risco? }\end{array}$ & 46,0 & 35,3 & 45,8 & 48,9 & 49,5 & 37,4 & $<0,001$ \\
\hline $\begin{array}{l}\text { Registra os casos mais graves em } \\
\text { saúde mental? }\end{array}$ & 52,9 & 33,2 & 50,7 & 62,4 & 54,6 & 36,3 & $<0,001$ \\
\hline $\begin{array}{l}\text { Registra os usuários de álcool e } \\
\text { outras drogas? }\end{array}$ & 36,8 & 15,0 & 33,7 & 46,0 & 41,5 & 21,2 & $<0,001$ \\
\hline $\begin{array}{l}\text { Registra os usuários em uso crônico de } \\
\text { psicotrópicos? }\end{array}$ & 55,0 & 42,5 & 55,6 & 56,4 & 58,4 & 51,0 & $<0,001$ \\
\hline $\mathrm{N}$ & 29.778 & 2.160 & 10.768 & 10.100 & 4.509 & 2.241 & \\
\hline
\end{tabular}

Fonte: Banco de dados do PMAQ-AB, segundo ciclo, 2013/2014.

Ver em:<http://dab.saude.gov.br/portaldab/ape_pmaq.php?conteudo=2_ciclo $>$.

A Tabela 3 congrega as ações de saúde mental agrupadas para uma avaliação global das dimensões priorizadas no estudo. Observou-se que $52,1 \%$ das equipes não realizavam ações de promoção de saúde mental. Apesar das diferenças observadas entre as regiões, o percentual de equipes que não realizavam nenhuma das ações de promoção foi elevado em todo o país. No que diz respeito à oferta de cuidados em saúde mental, somente 8,8\% das equipes relataram não ofertar quaisquer cuidados. A comparação entre as regiões evidenciou grande desigualdade: enquanto $2,9 \%$ das equipes da região Sul afirmaram não ofertar ações de cuidado em saúde mental, no Norte esse percentual foi de 22,6\%. Tal desigualdade se repetiu no campo gestão, em que $26,4 \%$ das equipes do Norte informaram não programar ações, em contraponto às regiões Sudeste e Sul, onde esse percentual caiu para $8,2 \%$ e $10,3 \%$, respectivamente.

Tabela 3

Dimensões da organização do cuidado em saúde mental pelas equipes de saúde da família no PMAQ-AB segundo ciclo. Brasil e regiões, 2013/2014

\begin{tabular}{|c|c|c|c|c|c|c|c|}
\hline \multirow[b]{2}{*}{ Dimensões } & \multirow[b]{2}{*}{ Brasil } & \multicolumn{5}{|c|}{ Região } & \multirow[b]{2}{*}{ p-valor } \\
\hline & & Norte & Nordeste & Sudeste & Sul & Centro-Oeste & \\
\hline Promoção de saúde mental & & & & & & & $<0,001$ \\
\hline 0 & 52,1 & 58,4 & 52,6 & 52,7 & 45,8 & 53,9 & \\
\hline 1-2 ações & 33,1 & 31,3 & 32,3 & 32,7 & 36,5 & 33,7 & \\
\hline 3 ações & 14,8 & 10,2 & 15,1 & 14,7 & 17,7 & 12,4 & \\
\hline
\end{tabular}


Continuação - Tabela 3

\begin{tabular}{|c|c|c|c|c|c|c|c|}
\hline \multirow[b]{2}{*}{ Variável } & \multirow[b]{2}{*}{ Brasil } & \multicolumn{5}{|c|}{ Região } & \multirow[b]{2}{*}{ p-valor } \\
\hline & & Norte & Nordeste & \multirow[t]{2}{*}{ Sudeste } & \multirow[t]{2}{*}{ Sul } & Centro-Oeste & \\
\hline Oferta de cuidado em & & & & & & & $<0,001$ \\
\hline \multicolumn{8}{|l|}{ saúde mental } \\
\hline 0 & 8,8 & 22,6 & 10,5 & 6,0 & 2,9 & 12,1 & \\
\hline 1-3 ações & 39,8 & 48,1 & 40,8 & 35,7 & 36,6 & 51,6 & \\
\hline 4-6 ações & 41,9 & 27,0 & 40,8 & 46,2 & 46,9 & 32,0 & \\
\hline 7 ações & 9,5 & 2,2 & 7,9 & 12,1 & 13,5 & 4,2 & \\
\hline Gestão do cuidado em & & & & & & & $<0,001$ \\
\hline \multicolumn{8}{|l|}{ saúde mental } \\
\hline 0 & 12,8 & 26,4 & 14,0 & 8,2 & 10,3 & 19,5 & \\
\hline 1-3 ações & 35,7 & 43,2 & 37,9 & 29,2 & 36,7 & 44,9 & \\
\hline 4-7 ações & 41,7 & 27,9 & 40,4 & 48,7 & 41,1 & 31,5 & \\
\hline 8 ações & 9,8 & 2,5 & 7,7 & 13,9 & 11,9 & 4,1 & \\
\hline $\mathrm{N}$ & 29.778 & 2.160 & 10.768 & 10.100 & 4.509 & 2.241 & \\
\hline
\end{tabular}

Fonte: Banco de dados do PMAQ-AB, segundo ciclo, 2013/2014.

Ver em:<http://dab.saude.gov.br/portaldab/ape_pmaq.php?conteudo=2_ciclo $>$.

A Tabela 4 mostra que a maior proporção de profissionais com preparo para lidar com as demandas de saúde mental se deu entre os que participavam de atividades de educação permanente. Em que pesem tais afirmações, o percentual de equipes que desempenhavam menos da metade das ações avaliadas ainda foi elevado nesse grupo, embora em menor proporção do que a observada entre os que se diziam despreparados.

\section{Tabela 4}

Dimensões da organização do cuidado em saúde mental pelas equipes de saúde da família no PMAQ-AB segundo ciclo. Brasil e regiões, 2013/2014

\begin{tabular}{|c|c|c|c|}
\hline \multirow{2}{*}{ Variáveis } & \multicolumn{2}{|c|}{ Educação permanente } & \multirow{2}{*}{ p-valor } \\
\hline & Sim & Não & \\
\hline Tem preparo para lidar com demandas em saúde mental? & & & $<0,001$ \\
\hline Sim & 42,5 & 17,6 & \\
\hline Não & 57,5 & 82,4 & \\
\hline Oferta de cuidado em saúde mental & & & $<0,001$ \\
\hline 0 & 7,9 & 16,4 & \\
\hline 1-3 ações & 37,9 & 55,2 & \\
\hline 4-6 ações & 43,8 & 26,5 & \\
\hline 7 ações & 10,4 & 1,9 & \\
\hline Gestão do cuidado em saúde mental & & & $<0,001$ \\
\hline 0 & 11,2 & 25,5 & \\
\hline 1-3 ações & 34,3 & 47,1 & \\
\hline 4-7 ações & 43,7 & 25,6 & \\
\hline 8 ações & 10,8 & 1,8 & \\
\hline $\mathrm{N}$ & & & 29.778 \\
\hline
\end{tabular}

Fonte: Banco de dados do PMAQ-AB, segundo ciclo, 2013/2014.

Ver em:<http://dab.saude.gov.br/portaldab/ape_pmaq.php?conteudo=2_ciclo $>$. 


\section{Discussão dos dados}

As equipes avaliadas eram, em grande parte, formadas por profissionais com até um ano de atuação na equipe. A precariedade de vínculo foi observada em equipes em todo o Brasil, exceto na região Sul; porém, nem a variável tempo nem o vínculo exerceram influência significativa nos itens investigados. Grande número de equipes $(88,2 \%)$ relatou atender a demandas de saúde mental, porém pouco mais de um terço afirmou estar preparado para lidar com esse tipo de demanda. Com relação à oferta de cuidado, as ações investigadas obtiveram índices baixos, à exceção do atendimento com profissionais de saúde mental, via Nasf ou outro dispositivo, que foi referido por $62 \%$ dos entrevistados. Esses percentuais elevados podem indicar que profissionais desses dispositivos estejam atuando de forma substitutiva, ou seja, atendendo eles mesmos os pacientes, em vez de atuarem como apoio matricial (Rodrigues e Moreira, 2012; Figueiredo e Campos, 2009).

O registro dos casos, elemento fundamental para a avaliação de quaisquer ações de saúde, mostrou-se uma ferramenta de uso incipiente nas regiões Norte e Centro-Oeste, o que impacta negativamente a gestão do cuidado aos casos. Já as regiões Sudeste e Sul, que despontam com os melhores resultados de registro em saúde (Souza, 2016), são também aquelas com maiores percentuais de formação complementar e com maior número de entrevistados que declararam preparo para lidar com demandas de saúde mental, sugerindo que a qualificação das equipes exerce influência positiva na gestão do cuidado. No tocante a esse item, chama a atenção o fato de que menos de $50 \%$ dos entrevistados afirmaram ter acesso aos protocolos clínicos e de classificação de risco. Ao se considerar que o registro dos usuários com demandas em saúde mental era realizado por pouco mais de metade das equipes, é possível inferir que parte desses profissionais vem atendendo e registrando os casos, sem contar com apoio de protocolos de orientação.

Os dados evidenciaram que grande parte dos profissionais da atenção básica não se sentia preparada para lidar com as demandas de saúde mental, situação identificada também em estudos anteriores (Tanaka e Ribeiro, 2009; Koga, Furageto e Santos, 2006). A relatada falta de preparo vem sendo atribuída (Rodrigues e Moreira, 2012; Tanaka e Ribeiro, 2009) a uma formação profissional que não capacita para a atenção dos problemas de saúde mental na atenção básica, ou a um entendimento equivocado de que a saúde mental só pode ser trabalhada pelos especialistas da área (Rodrigues e Moreira, 2012).

As ações educativas e de promoção de saúde em saúde mental obtiveram pouco mais de um terço das respostas afirmativas, revelando baixa incorporação dessas atividades na rotina dos serviços e distanciamento das recomendações da Política Nacional de Atenção Básica (PNAB). Baixos índices de execução 
de ações de promoção de saúde não são exclusividade do campo de saúde mental, havendo estudos (Garnelo et al., 2014; Medina et al., 2014) que confirmam a incipiência das ações de promoção e prevenção a doenças crônicas em geral no nível primário. Teixeira e colaboradores (2014) também apontaram que nas regiões Norte, Nordeste e Centro-Oeste o índice de organização de ações educativas e de promoção da saúde era muito menor que na ESF do Sudeste e do Sul, onde mais de $80 \%$ das equipes organizavam a agenda para oferecer essas atividades.

Outras ações com baixa incorporação na rotina são aquelas voltadas para usuários de álcool e outras drogas e medicamentos psicotrópicos, que obtiveram índices baixos tanto na oferta de cuidado quanto na gestão. A esse respeito, Koga, Furageto e Santos (2006) também constataram que profissionais da ESF em Maringá (PR) não tinham preparo para orientar os usuários sobre o uso de medicamentos psicotrópicos. Estudos do primeiro ciclo do PMAQ igualmente vêm apontando baixa implementação de ações de prevenção do abuso de psicotrópicos e de promoção da saúde mental (Teixeira et al., 2014).

A Política Nacional Antidrogas ressalta a importância do desenvolvimento de ações no plano local onde se efetiva o risco (Brasil, 2002), colocando em destaque o papel da equipe de saúde da família, cujo conhecimento abrangente da realidade do território e cuja inserção na comunidade desempenham papel fundamental na prevenção ao uso de drogas e no favorecimento de intervenções contínuas e de base territorial.

O uso abusivo de substâncias costuma envolver questões de ordem social, familiar e psicológica que devem ser abordadas em profundidade, o que demanda qualificação adequada dos profissionais, além de integração com a rede de referência para atendimento de casos mais graves (Figueiredo e Campos, 2009). Nesse sentido, a coordenação do cuidado pela equipe de saúde da família (eSF) assume papel crucial para garantir a qualidade da atenção. A constatação de que mais de $50 \%$ das equipes entrevistadas não efetuavam a gestão do cuidado e apresentavam baixa adesão ao uso de protocolos com diretrizes terapêuticas e de estratificação de risco dos usuários com transtornos mentais indicou limitada capacidade organizativa, o que compromete a efetividade e a integralidade das ações. Trata-se de um cenário já delineado em publicação de 2009 (Tanaka e Ribeiro, 2009), que parece não ter sofrido alterações.

O insuficiente preparo das eSFs para lidar com as demandas - não apenas de saúde mental - também tem sido observado em outros estudos (Souza e Scatena, 2007; Spadini e Souza, 2010), que apontam o predomínio dos saberes biomédicos na formação dos profissionais como fator impeditivo da aquisição de subsídios para o manejo dos aspectos psicológicos e emocionais, ao lado de uma educação permanente pouco direcionada para subsidiar a atividade 
prática (Tanaka e Ribeiro, 2009; Souza e Scatena, 2007; Arantes, Shimizu e Mérchan-Hamann, 2016).

Outros aspectos associados, como a inadequada organização do processo de trabalho, com consultas curtas, falta de medicamentos, inexistência ou dificuldade de articulação com a rede de apoio e baixo retorno dos encaminhamentos (Tanaka e Ribeiro, 2009; Spadini e Souza, 2010; Arantes, Shimizu e Mérchan-Hamann, 2016), coexistem com uma rápida expansão da ESF sem o equivalente aporte da rede de referência (Tanaka e Ribeiro, 2009), o que contribui para agravar o panorama descrito. Os dados aqui analisados sugerem que as ações de educação permanente influenciam na maior oferta de cuidado e no aprimoramento da gestão local que visa garanti-la; entretanto, não foram suficientes para elevar os índices das equipes para além de 50\% de realização.

A análise do manejo das demandas dos usuários evidenciou baixa oferta de atendimento em grupos, remetendo ao despreparo mencionado pelas equipes identificado também por Spadini e Souza (2010). O trabalho em grupo é estratégia prioritária para a promoção de saúde (Moliner e Lopes, 2013; Nogueira et al., 2016) e para a abordagem de portadores de transtornos mentais, sendo uma técnica frequentemente utilizada pelos profissionais que realizam apoio matricial (Campos, 1999), ou seja, os de maior qualificação para atender a saúde mental.

\section{Considerações finais}

O conjunto de resultados sugere que a inserção da saúde mental na atenção básica é incipiente; que há expressiva fragilidade na oferta organizada do cuidado; baixo registro dos casos; ausência de protocolos nas unidades; limitado desenvolvimento de ações de promoção de saúde e para usuários de álcool, medicamentos psicotrópicos e outras drogas. Tais problemas prejudicam a definição dos fluxos e agravam a insuficiência da gestão do cuidado em saúde mental. Há lacunas com relação à formação dos profissionais. Estas devem ser preenchidas mediante a universalização do acesso aos protocolos e às técnicas que permitam identificar, estratificar e abordar com segurança os problemas de saúde mental. Os níveis centrais de gestão do Sistema Único de Saúde (SUS) também precisam definir protocolos e indicadores - hoje inexistentes - que estabeleçam perfis e parâmetros de atendimento para esse público na atenção básica.

O crescimento da prevalência de condições crônicas no perfil epidemiológico da população brasileira, aí incluídos os transtornos mentais, exige melhoria na qualificação do cuidado e fortalecimento da rede de atenção psicossocial. A articulação entre a saúde mental e a atenção básica é ainda frágil e seu desenvolvimento muito desigual no país, acompanhando o perfil das 
iniquidades regionais, seja no que diz respeito às condições de vida, seja na distribuição da rede assistencial. Atenção particular deve ser dada à região Norte, onde limitações na distribuição e qualidade nos serviços ofertados somam-se às dificuldades de acesso geográfico, o que confere um papel ainda mais estratégico à atuação das equipes de saúde da família.

Como principais limitações do estudo, aponta-se o fato de a amostra não ser aleatória e sim resultante da adesão voluntária das equipes. Esta precondição é amenizada pelo alcance de $87,1 \%$ das equipes que atuam no país. Ainda assim, persiste um viés de seleção, já que os municípios tenderiam a fazer adesão de suas equipes com melhor desempenho, com consequente elevação das respostas positivas (Fausto et al., 2014). Esta condição recomenda cautela na generalização dos resultados, pois, caso disponíveis, as respostas das equipes que não aderiram poderiam evidenciar resultados ainda piores.

O instrumento de coleta de dados do PMAQ também carece de precisão na formulação de alguns quesitos, como na caracterização de subgrupos investigados (usuários de drogas e de medicamentos psicotrópicos) e de algumas ações ofertadas ("Registra casos mais graves em saúde mental?"), o que dificulta o adensamento das discussões sobre a temática. Apesar dessas limitações, a análise dos resultados da avaliação externa propiciou um amplo retrato da oferta desse tipo de cuidado na $\mathrm{AB}$, identificando necessidades de fortalecimento da rede e de qualificação das equipes de saúde da família para a atuação em saúde mental.

\section{Colaboradores}

Tiziana Bezerra Gerbaldo, Adenilda Teixeira Arruda e Bernardo Lessa Horta atuaram na concepção do projeto, análise dos dados e redação final do artigo. Luiza Garnelo atuou na concepção do projeto, coleta e análise dos dados e redação final do artigo. 
Resumen Este artículo resultó de un estudio transversal sobre el cuidado de la salud mental realizado con 29.778 equipos de la Estrategia de Salud de la Familia de todo Brasil $(87,1 \%$ del total), incluyendo la evaluación normativa de los datos del segundo ciclo del Programa Nacional de Mejora del Acceso y Calidad de la Atención Básica (2013-2014) en las dimensiones perfil de los profesionales, promoción de la salud mental, gestión y oferta del cuidado. Los resultados demostraron que el 33,8\% de los entrevistados contaban con una relación laboral precaria y el $60,3 \%$ de ellos no se sentía preparado para trabajar en la salud mental. La oferta del conjunto de acciones evaluadas sólo se produjo en el 9,5\% de los equipos de todo Brasil $(2,2 \%$ en la región Norte). Alrededor de la mitad no desarrollaba estrategias de promoción de la salud y sólo el 9,8\% hacía efectiva la gestión del cuidado. Se concluyó que los bajos porcentajes de implantación a nivel nacional coexisten con expresivas desigualdades regionales, con peores resultados en el Norte. Se hace necesario el fortalecimiento de acciones de promoción de la salud, la cualificación de los equipos, la desprecarización de las relaciones laborales y la reordenación de la gestión del cuidado.

Palabras clave salud mental; atención básica; programas de salud.

\section{Notas}

${ }^{1}$ Fundação Oswaldo Cruz, Centro de Pesquisa Leônidas e Maria Deane, Programa de Pós-Graduação em Condições de Vida e Situações de Saúde na Amazônia, Manaus, Amazonas, Brasil.

$<$ tiziana.gerbaldo@gmail.com>

Correspondência: Rua 17, número 267, Conjunto dos Subtenentes, Flores, CEP 69028-416, Manaus, Amazonas, Brasil.

${ }^{2}$ Universidade Federal do Amazonas, Pró-Reitoria para Assuntos Comunitários, Manaus, Amazonas, Brasil.

$<$ adenildaarruda@gmail.com>

${ }^{3}$ Universidade Federal de Pelotas, Faculdade de Medicina, Departamento de Medicina Social, Pelotas, Rio Grande do Sul, Brasil.

<blhorta@gmail.com>

${ }^{4}$ Fundação Oswaldo Cruz, Centro de Pesquisa Leônidas e Maria Deane, Manaus, Amazonas, Brasil.

<luiza.garnelo@amazonia.fiocruz.br>

${ }^{5} \mathrm{O}$ estudo foi resultado da dissertação de mestrado Organização do cuidado em saúde mental no Brasil: uma análise a partir da avaliação externa do PMAQ-AB, de autoria de Tiziana Bezerra Gerbaldo, apresentada ao Programa de Pós-Graduação em Condições de Vida e Situações de Saúde na Amazônia, do Instituto Leônidas e Maria Deane, Fiocruz Amazônia, em 30/08/2017. Ver em: <https://www.arca.fiocruz.br/handle/icict/26015>. 


\section{Referências}

ARANTES, Luciano J.; SHIMIZU, Helena E.; MERCHÁN-HAMANN, Edgar. Contribuições e desafios da Estratégia Saúde da Família na atenção primária à saúde no Brasil: revisão de literatura. Ciência \& Saúde Coletiva, Rio de Janeiro, v. 21, n. 5, p. 1.499-1.510, 2016.

BRASIL. Ministério da Justiça. Secretaria Nacional Antidrogas. Política Nacional Antidrogas. Decreto n. 4.345, de 26 de agosto de 2002. Brasília, 2002. Disponível em: < http://www. planalto.gov.br/ccivil_03/decreto/2002/d4345. htm >. Acesso em: 5 fev. 2016.

BRASIL. Ministério da Saúde. Saúde mental e atenção básica: o vínculo e o diálogo necessários, inclusão das ações de saúde mental na atenção básica. Brasília: Ministério da Saúde, 2003. Disponível em: <https://www.nescon. medicina.ufmg.br/biblioteca/imagem/1734. pdf $>$. Acesso em: 8 fev. 2016.

BRASIL. Ministério da Saúde. Portaria MS/ GM n. 3.088, de 23 de dezembro de 2011. Institui a Rede de Atenção Psicossocial para pessoas com sofrimento ou transtorno mental e com necessidades decorrentes do uso de crack, álcool e outras drogas, no âmbito do Sistema Único de Saúde. Brasília: Ministério da Saúde, 2011. Disponível em: < http:// bvsms.saude.gov.br/bvs/saudelegis/gm/2011/ prt3088_23_12_2011_rep.html>. Acesso em: 10 dez. 2015.

BRASIL. Ministério da Saúde. Política Nacional de Atenção Básica. Brasília: Ministério da Saúde, 2012. Disponível em: <http://189.28.128.100/ $\mathrm{dab} /$ docs/publicacoes/geral/pnab.pdf $>$. Acesso em: 12 nov. 2015.

BRASIL. Ministério da Saúde. Manual instrutivo para as equipes de atenção básica (saúde da família, saúde bucal e equipes parametrizadas) e Nasf. Brasília: Ministério da Saúde, 2013. Disponível em: <http://189.28.128.100/ dab/docs/portaldab/publicacoes/manual_instrutivo_PMAQ_AB2013.pdf>. Acesso em: 24 out. 2015.
BRASIL. Ministério da Saúde. Secretaria de Atenção à Saúde. Departamento de Regulação, Avaliação e Controle de Sistemas. Critérios e parâmetros para o planejamento e programação de ações e serviços de saúde no âmbito do Sistema Único de Saúde. Brasília: Ministério da Saúde, 2015. Disponível em: <http://www.fehosp.com.br/ app/webroot/files/manuais/5ffa8dle03f7edb0le leed7b07178cfb.pdf>. Acesso em: 6 mar. 2016.

BRASIL. Ministério da Saúde. Departamento de Atenção Básica. Teto, credenciamento e implantação das estratégias de agentes comunitários de saúde, saúde da família e saúde bucal. Brasil, jan. 2013 a dez. 2014. Tabela. Brasília: Ministério da Saúde, 2016. Disponível em: <http://dab.saude. gov.br/portaldab/historico_cobertura_sf.php $>$. Acesso em: 3 jun. 2016.

CAMPOS, Gastão W. S. Equipes de referência e apoio especializado matricial: um ensaio sobre a reorganização do trabalho em saúde. Ciência \& Saúde Coletiva, Rio de Janeiro, v. 4, n. 2, p. 393-403, 1999.

CECÍLIO, Luiz C. O. Apontamentos teóricoconceituais sobre processos avaliativos considerando as múltiplas dimensões do cuidado em saúde. Interface: Comunicação, Saúde e Educação, Botucatu, v. 15, n. 37, p. 589-599, 2011.

CHAMPAGNE, François et al. A apreciação normativa. In: BROUSSELLE, Astrid et al. Avaliação: conceitos e métodos. Rio de Janeiro: Editora Fiocruz, 2011. p. 77-94.

FAUSTO, Márcia C. R. et al. A posição da Estratégia Saúde da Família na rede de atenção à saúde na perspectiva das equipes e usuários participantes do PMAQ-AB. Saúde em Debate, Rio de Janeiro, v. 38, p. 13-33, out. 2014. Edição especial.

FIGUEIREDO, Mariana D.; CAMPOS, Rosana O. Saúde mental na atenção básica à saúde de Campinas, SP: uma rede ou um emaranhado? Ciência \& Saúde Coletiva, Rio de Janeiro, v. 14, n. 1, p. 129-138, 2009. 
GARNELO, Luíza et al. Organização do cuidado às condições crônicas por equipes de saúde da família na Amazônia. Saúde em Debate, Rio de Janeiro, v. 38, p. 158-172, out. 2014. Edição especial.

KOGA, Mariko; FURAGETO, Antonia R. F.; SANTOS, Jair L. F. Opiniões da equipe e usuários sobre a atenção à saúde mental num programa de saúde da família. Revista Latino-Americana de Enfermagem, Ribeirão Preto, v. 14, n. 2, p. 163-169, mar.-abr. 2006.

MARAGNO, Luciana et al. Prevalência de transtornos mentais comuns em populações atendidas pelo Programa Saúde da Família (Qualis) no município de São Paulo, Brasil. Cadernos de Saúde Pública, Rio de Janeiro, v. 22, n. 8, p. 1.639-1.648, ago. 2006.

MEDINA, Maria G. et al. Promoção de saúde e prevenção de doenças crônicas: o que fazem as equipes de saúde da família? Saúde em Debate, Rio de Janeiro, v. 38, p. 69-82, out. 2014. Edição especial.

MOLINER, Juliane; LOPES, Stella M. B. Saúde mental na atenção básica: possibilidades para uma prática voltada para a ampliação e integralidade da saúde mental. Saúde $e$ Sociedade, São Paulo, v. 22, n. 4, p. 1.0721.083, dez. 2013.

MOREIRA, Juliana K. P. et al. Prevalência de transtornos mentais comuns e fatores associados em uma população assistida por equipes do Programa Saúde da Família. Jornal Brasileiro de Psiquiatria, Rio de Janeiro, v. 60, n. 3, p. 221-226, 2011.

NOGUEIRA, Alyne L. G. et al. Pistas para potencializar grupos na atenção primária à saúde. Revista Brasileira de Enfermagem, Brasília, v. 69, n. 5, p. 964-971, out. 2016.

RODRIGUES, Euzilene S.; MOREIRA, Maria I. B. A interlocução da saúde mental com atenção básica no município de Vitória. Saúde e Sociedade, São Paulo, v. 21, n. 3, p. 599-611, 2012.
SCHOENBAUM, Michael et al. The effects of primary care depression treatment on patient's clinical status and employment. Health Services Research Journal, Chicago, v. 37, n. 5, p. 1.145-1.158, out. 2002.

SOUZA, Maximiliano L. P. Registro de distúrbios mentais no Sistema de Informação da Atenção Básica do Brasil, 2014. Epidemiologia e Serviços de Saúde, Brasília, v. 25, n. 2, p. 405-410, 2016.

SOUZA, Rozemere C.; SCATENA, Maria C. M. Possibilidades e limites do cuidado dirigido ao doente mental no programa de saúde da família. Revista Baiana de Saúde Pública, Salvador, v. 31, n. 1, p. 147-160, 2007.

SPADINI, Luciene S.; SOUZA, Maria C. B. M. O preparo de enfermeiros que atuam em grupos na área de saúde mental e psiquiatria. Escola Anna Nery Revista de Enfermagem, Rio de Janeiro, v. 14, n. 2, p. 355-360, 2010.

TANAKA, Oswaldo U.; RIBEIRO, Edith L. Ações de saúde mental na atenção básica: caminho para ampliação da integralidade da atenção. Ciência \& Saúde Coletiva, Rio de Janeiro, v. 14, n. 2, p. 477-486, 2009.

TEIXEIRA, Mirna B. et al. Avaliação das práticas de promoção de saúde: um olhar das equipes participantes do Programa Nacional de Melhoria do Acesso e da Qualidade da Atenção Básica. Saúde em Debate, Rio de Janeiro, v. 38, p. 52-68, out. 2014. Edição especial.

WORLD HEALTH ORGANIZATION (WHO). The World Health Report: mental health new understanding, new hope. Washington: World Health Organization, 2001.

WORLD HEALTH ORGANIZATION (WHO). Mental Health Gap Programme: scaling up care for mental, neurological and substance disorders. Washington: World Health Organization, 2008.

Recebido em 07/08/2017.

Aprovado em 19/01/2018. 